\title{
Transient high density vertebral bone lesions
}

\author{
Chakib Alili • Ahmed Larbi • Yann Thouvenin • \\ Pierre Viala • Alban Ruyer • Marie-Pierre Baron • \\ Catherine Cyteval
}

Published online: 10 May 2013

(C) ISS 2013

\section{Diagnosis}

Bone marrow enhancement by retrograde contrast filling through collateral veins due to a left brachiocephalic vein thrombosis.

\section{Discussion}

The contrast-enhanced CT examination revealed sclerotic-like lesions associated with consecutive anterior and posterior elements from the fourth cervical vertebra to the fifth thoracic vertebra, without any lytic components, which could at first be considered as bony metastases (Fig. 1a-b). CT scan also highlighted significant collateral venous circulation secondary to a brachiocephalic left venous thrombosis. A PET-CT performed at the same time was negative. A non-contrast CT scan performed 10 days later did not reveal the initially noted bone lesions (Fig. 2a-b) and suggested diagnosis of intraosseous venous contrast media. The 1-year non-contrast CT follow-up did not show any progression of the disease.

Given the lack of corresponding findings on the unenhanced CT, in the setting of central venous obstruction and collateral pathway formation, the high-attenuation lesions seen on the enhanced CT examination can be explained by the presence of intraosseous venous collaterals. In fact, the left brachiocephalic vein obstruction was responsible for the development of an extensive collateral venous pathway (Fig. 2c-d) involving the mediastinum, anterior jugular veins, anterior, and basivertebral venous plexi. The venous pressure

The case presentation can be found at doi: 10.1007/s00256-013-1619-2

C. Alili $(\bowtie) \cdot$ A. Larbi $\cdot$ Y. Thouvenin $\cdot$ P. Viala $\cdot$ A. Ruyer

M.-P. Baron - C. Cyteval

Department of Radiology CHU Lapeyronie, Montpellier, France

e-mail: chakibalili@yahoo.fr in the dilated capillaries allowed marrow enhancement through the anastomosis capillary sites: basivertebral veins bed (between the anterior external plexus and the posterior internal plexi) and pedicular sites (between the intervertebral veins and the vertebral plexi) (Fig. 3a-b). Our patient remained asymptomatic despite the huge collateral venous substitution. The rate of injection of contrast and the time of image acquisition following contrast injection might be factors influencing the appearance of these findings.

Chest collateral veins usually occur in superior vena cava obstruction and then develop through four classic pathways: azygos and hemiazygos, internal thoracic and laterothoracic, superficial thoracoabdominal, and vertebral venous plexus (posterior way) $[1,2]$. These patients usually present with a symptomatic superior vena cava syndrome [3]. Less frequently, chest collateral veins can develop secondary to unilateral thrombosis of the brachiocephalic venous trunk, as in our case where the posterior pathway was involved [4].

Several cases of visceral abnormalities related to superior vena cava syndrome, including pericardial and hepatic enhancement, have been described [5]. However, bone involvement is uncommon. Only, two cases of cervical vertebral bone high-density abnormalities have been described in the context of superior vena cava syndrome secondary to a mediastinal mass (epidermoid lung carcinoma and small-cell lung carcinoma) [6, 7]. To our knowledge, there are no reports of similar cases of vertebral marrow abnormalities in asymptomatic patients, without any mass syndrome or obstructed superior cava vein.

In the case presented, the findings could be misinterpreted as lymphomatous bone marrow involvement because of the patient's history even though bony features of osseous lymphoma are usually lytic and seldom sclerotic, with soft tissue involvement.

In summary, the present case illustrates a benign lesion entity related to retrograde contrast filling in bone marrow. 


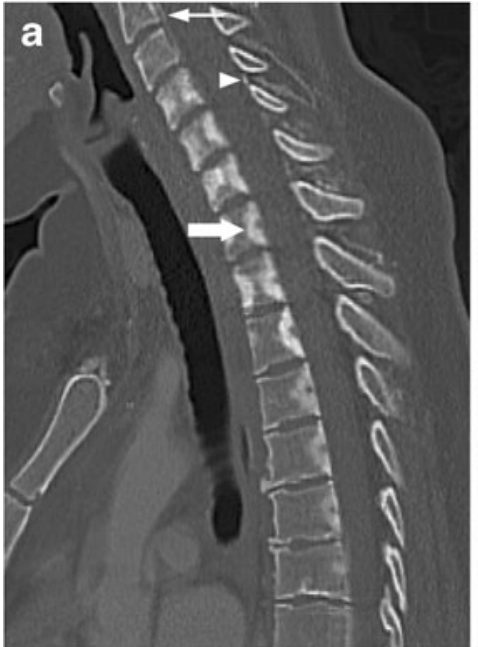

Fig. 1 a Contrast-enhanced thoracic CT (sagittal view) showing focal high-density marrow lesion consecutively concerning the anterior and posterior $\mathrm{C} 4$ to $\mathrm{Th} 5$ vertebral bodies. Marrow enhancement corresponded to venous plexi anastomosis sites: anterior and posterior basivertebral venous bed (large arrow), filled from the internal venous

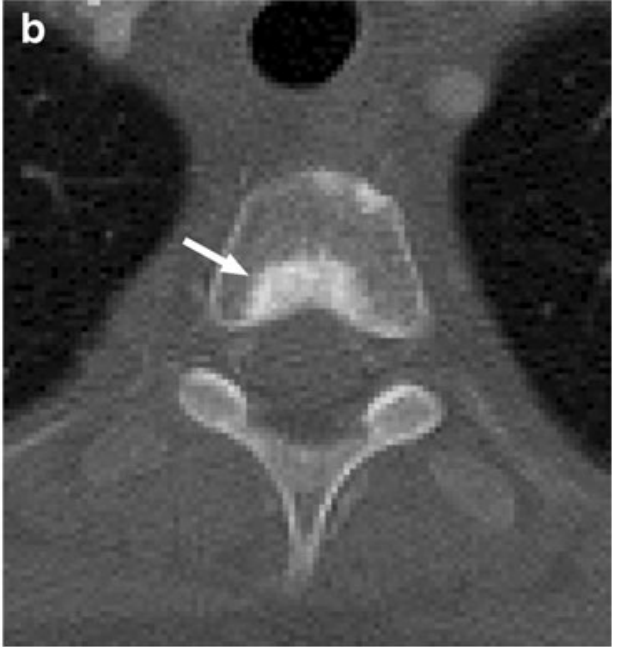

plexus (thin arrow) and intervertebral veins (arrowhead). b Contrastenhanced thoracic CT (axial view) demonstrating increased density within the upper thoracic vertebral body in the basivertebral venous bed, without mass in soft tissues
Fig. 2 Non-contrast CT scan performed 10 days after showing regression of the lack of findings to correspond to those seen on the initial contrast-enhanced exam on the sagittal view (a) and axial view (b). c Extensive anterior and posterior chest collateral veins. d Left brachiocephalic vein thrombosis (arrow)
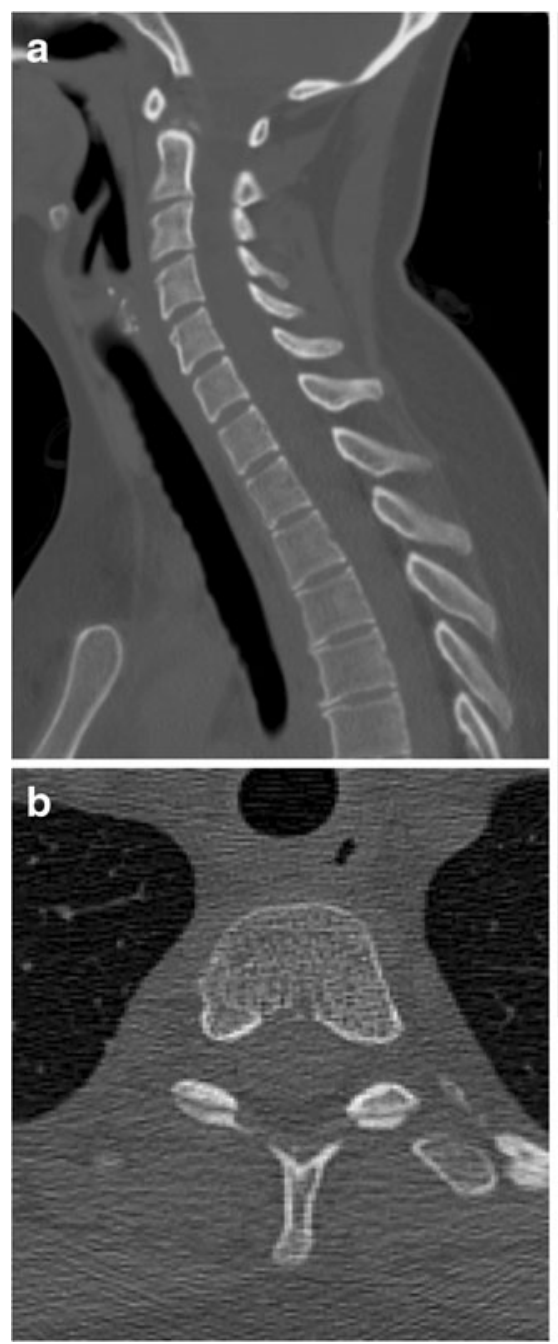

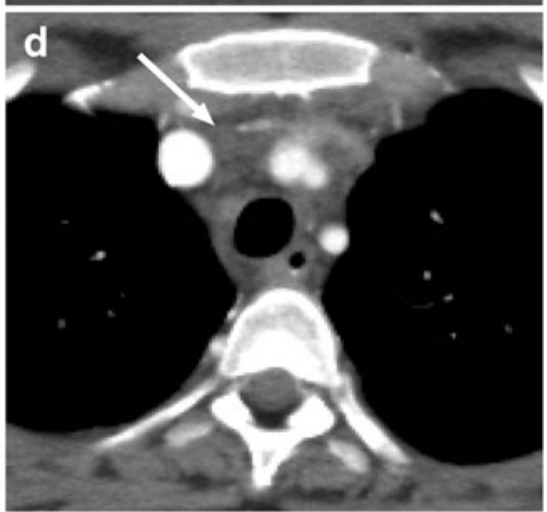




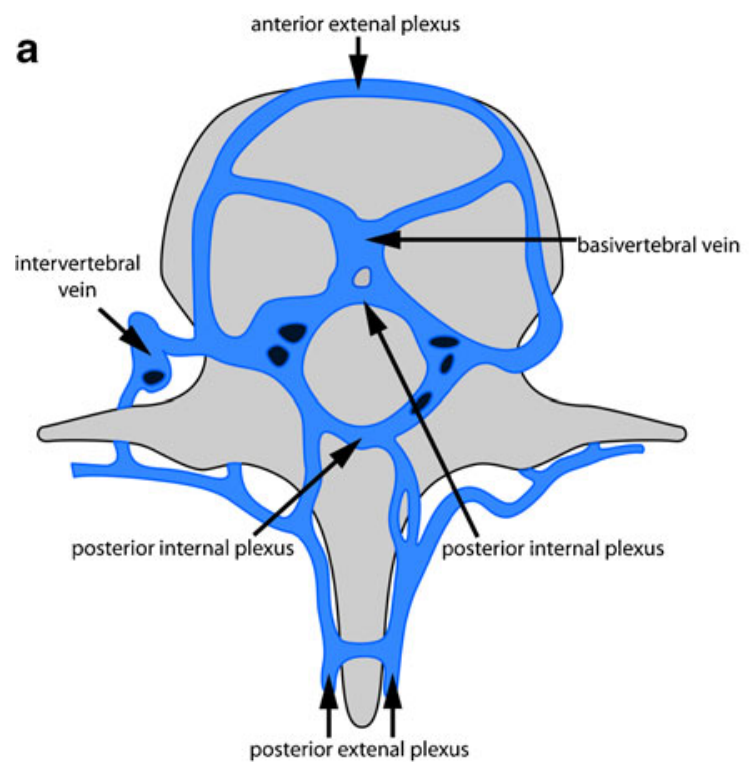

Fig. 3 Axial (a) and sagittal (b) view showing the vertebral posterior network through the different venous plexi, presenting as a large avalvular system, thus explaining the high-density lesion distribution:

When performing a contrast-enhanced CT revealing a thoracic venous obstruction with collateral veins, high-density bone entity due to the presence contrast within the vertebral body veins have to be differentiated from progressive metastatic bone involvement.

\section{References}

1. Eckhenhoff JE. The vertebral venous plexus. Can J Anaesth. 1971;18:487-95.

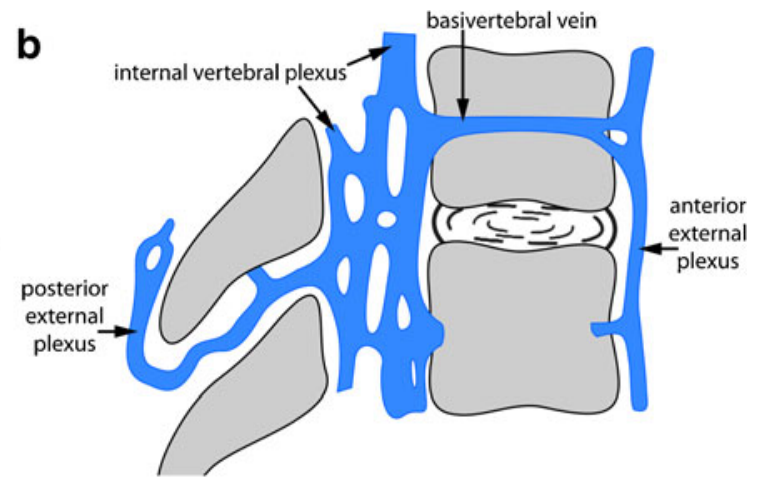

basi-vertebral vein bed (posterior corporeal anastomosis of the two plexi) and pediclar regions (lateral pediclar anastomosis though the intervertebral veins)

2. Sheth S, Ebert MD, Fishman EK. Superior vena cava obstruction evaluation with MDCT. Am J Roentgenol. 2010;194:W336-46.

3. Wilson ES. Systemic to pulmonary venous communication in the superior vena caval syndrome. Am J Roentgenol. 1976;127:247-9.

4. Parish JM, Marschke Jr RF, Dines DE, Lee RE. Etiologic considerations in superior vena cava syndrome. Mayo Clin Proc. 1981;56:407-13.

5. Holemans JA, Howlett DC, Rankin SC. Case report: superior vena cava obstruction: unusual CT findings due to venous collaterals. Clin Radiol. 1997;52:559-60.

6. Thomas N, Oliver TB, Sudarshan T. Vanishing bone metastases a pitfall in the interpretation of contrast enhanced $\mathrm{CT}$ in patients with superior vena cava obstruction. Br J Radiol. 2011;84:e176-8.

7. Jesinger RA, Huynch B, Gover D. Superior vena cava syndrome resulting in osseous venous congestion simulating sclerotic bone lesions. AJR 2009; 192:W344 0361 